\title{
Twitter Data Collection Using Tools for Classification of fake Data: A Survey
}

\author{
Mr. Aannd $\mathbf{R}^{1 *}(0000-0001-5028-8661)$, Mr. Muneshwara $\mathrm{MS}^{2}(0000-0003-4714-4100)$ \\ Dr. Deepak S Sakkari ${ }^{3}(0000-0001-5986-3626)$ \\ ${ }^{1,2}$ BMS Institute of Technology and Management, Yelahanka, Bangalore 560064, India. \\ ${ }^{3}$ School of Engineering, Presidency University, Rajanakunte, Yelahanka, Bengaluru 560064, India. \\ anandor@bmsit.in, muneshwarams@bmsit.in, deepakssakkariepres \\ idencyuniversity.in
}

\begin{abstract}
Data could be a piece of information that's needed to create helpful information. Getting data is required by people to analyzers. From this angle, data assortment is a vital step once doing any research or experiment. Knowledge collection may be outlined because the method of gathering and process the data to gauge the outcomes and use them for the researches. On-line Social Networking sites (OSN) are one in all the most effective sources of data. We have a tendency to be attending to introduce the advantages of exploitation the social network sites for data collection and also the totally different techniques which will be used. Based mostly on those data, a network of trust created exploitation the relationships among users. The methods the information being collected is totally different in term of potency and being useful. Be that as it may, the information mining applications in the web-based media are as yet crude and require more exertion by the scholarly world and industry to sufficiently play out the work. Client created content via online media destinations, for example, Twitter and Facebook gives freedom to specialists in different fields to comprehend human practices and social marvels. From one viewpoint, these human practices and social marvels are unpredictable in nature hence need top to bottom subjective investigation. On the other, the size of online media data requires colossal degree data assessment strategies. Automated information assortment of interpersonal interaction Web locales assumes a significant part in dynamic. Realize that the Web destinations like Twitter, Facebook, YouTube, Pin interest, and so on are turning out to be indispensable parts of public activity as of now. In any examination issue the mass effect on different issues can be investigated by breaking down the information produced from these Web locales. Also, these social stages are open and generally utilized for see sharing. Here different devices and systems have been assessed to gather the information from these Web locales. The capacities of conclusion investigation stretch out to the quantity of genuine choices like medical problems in the public eye, or the client responses, and so forth in this paper information assortment procedures have been shown with the assistance of live execution.
\end{abstract}

Keywords: On-line Social Networking, Twitter, Facebook, Web locales. 


\section{Introduction}

As of start of 2016 the quantity of enrolled clients in Facebook arrived at 1.7 billion clients, Facebook page was accessed by 1.1 billion people.. In the meantime the quantity of enlisted client's 392 million people used VK. According to Genius study findings for January 2016, 20.2 million clients in Ukraine basically once a month on work area or mobile phones/cell phones/tablets, and $70 \%$ of them visit the website www.vk.com.

Exploration life cycle includes various stages like arranging the proposition, beginning the venture, gathering the information, dissecting the information and getting the outcome. As should be obvious, information assortment plays a significant stage since it responds to the exploration questions.

Gathering information should be possible by utilizing various strategies dependent on the wellspring of the information. A few models identified with information sources affects individuals interviews, reviews disseminations and Social site destinations.

Because social sites retain information about people, their interactions with other on the same website, as well as Depending on the sort of site, their ranking, reviews, or even rating information, they a valuable source for data gathering. While gathering data, several considerations should be made, such as accuracy, dependability, and security.

- Precision: The correctness of the data that's gathered is critical since inaccurate data can outcome in erroneous study conclusions or observations. As a outcome, reliable data will be important in maintaining the research's integrity.

- Reliability: dependability of the data is considered from source to see it's dependable or not from this.

- Security: Because certain data is meant to be kept private, the privacy of the data must be considered. As a outcome, before collecting information, the user should determine if he or she is authorized for receive that. For instance, on Twitter, few user accounts are secret, and if the user gains access to them without authority, this constitutes an invasion of privacy. As illustrated in Fig, the acquired info may be analyzed and used to develop an app. Platforms where users may openly submit material are known as social sites. Data analysis aids in determining people's perceptions of how the product works. These websites serve as clubhouses where clients pay close attention to communication messages. This, in turn, assists marketers and customers in raising their levels. There are three distinct sorts of Web sites from which information should be extracted:

- Unstructured pages: Unstructured pages, often known as free-text papers, are in natural language. There is no discernible structure, and only information extraction (IE) approaches may be utilized confidently. 
- Structured pages: often received from a structured info source, as a database, and info is given with structural information. The data is extracted using simple approaches on matching.

- Semi-structured pages: a middle ground unstructured and structured pages differ in that they do not adhere to a definition of the sorts of data published on them. Papers have some structure in any case, and extraction approaches are frequently predicated on existence of specific design, such HTML tags amount of data that can be gleaned by these materials is quite restricted.

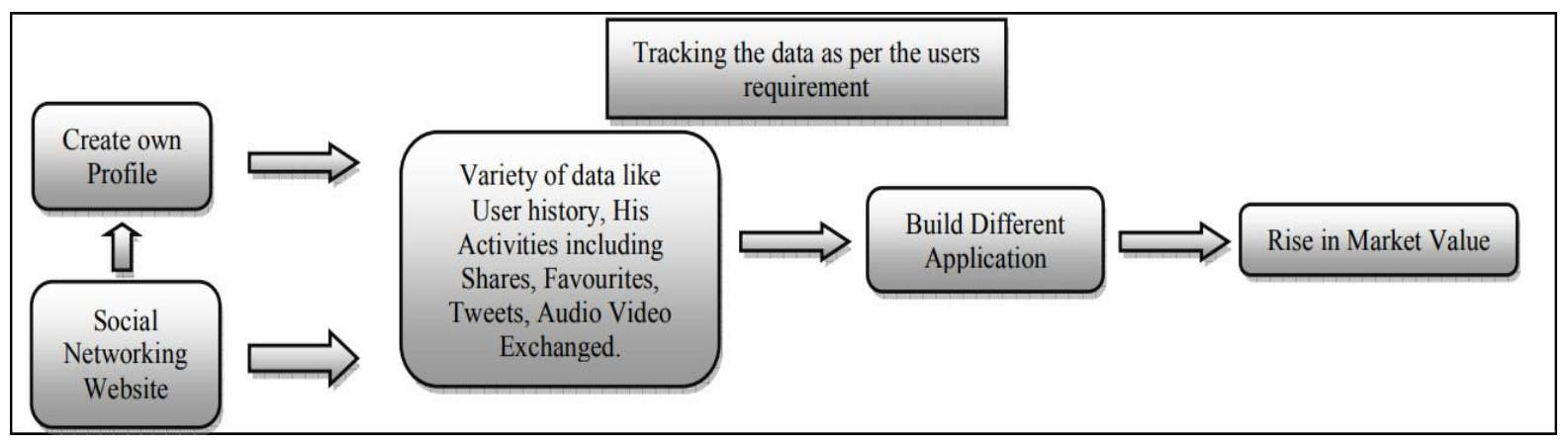

Figure1: Tracking the Data

\section{Existing System}

There have been several studies that have employed social site to obtain data. Instance, there is project devoted for comprehending Twitter. "When daily deal services meet Twitter: Understanding Twitter as a daily deal marketing platform," said "for this study, has a set of tweets has the URL of the 'groupon.com.

"A study of security in vehicle ad hoc networks using identity-based cryptography," info of Facebook was collected for utilizing Latent Dirichlet Allocation (LDA) to find themes of greater 500,000 Facebook updates and find what title are to generate response, ex.. likes. "This study reveals gender disparities in the themes of status updates on SNS," they added. ladies are inclined than men to mention for individual issues, whereas males are about issues. Gender differences are masked in adolescence".

A study has suggested demonstrating that a Trust System can give trust. Info gathering were required for conduct the tests and evaluate the outcomes. Twitter was an excellent data source for our study. The plan was to look for Twitter groups that discuss the stock market. It was designed to gather user IDs for subsequently use for obtaining data from Twitter. This will be shown in this article as example of data is gathered. 


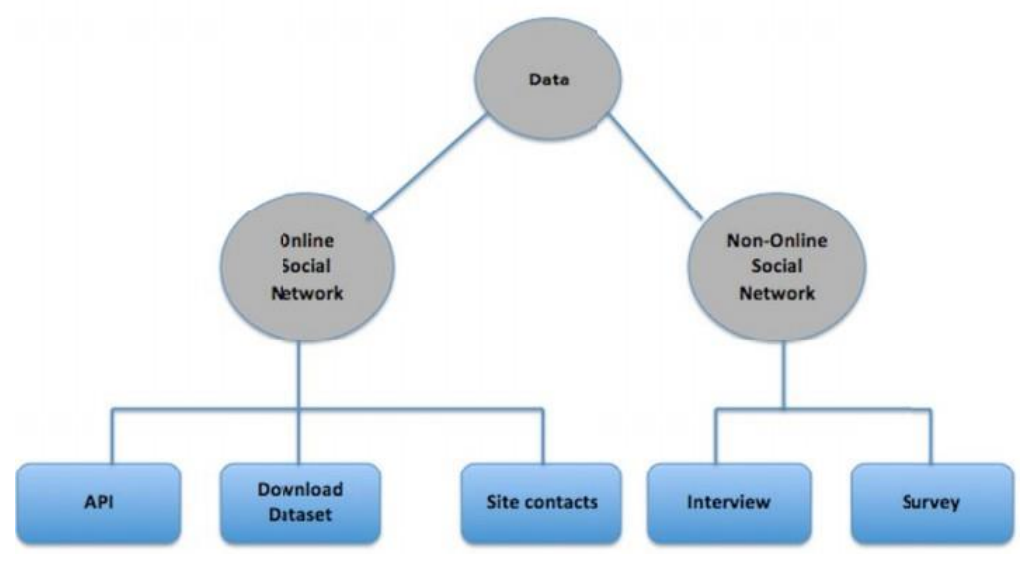

Figure 2: Online/Non-Online Data on Social Network

\section{Methodology}

We can have info from many social sites such as Twitter, Facebook, and yelp. Obtaining info may be accomplished in a variety of ways, including talking the administrator hold the data, getting the data developed for academic, or participating in a hurdle. Each social network has an API (Application Programming Interface) that allows the data user to request services from sites. Installing their relevant libraries, gaining authorization, and selecting site in which the user may write the code are s steps for such sites.

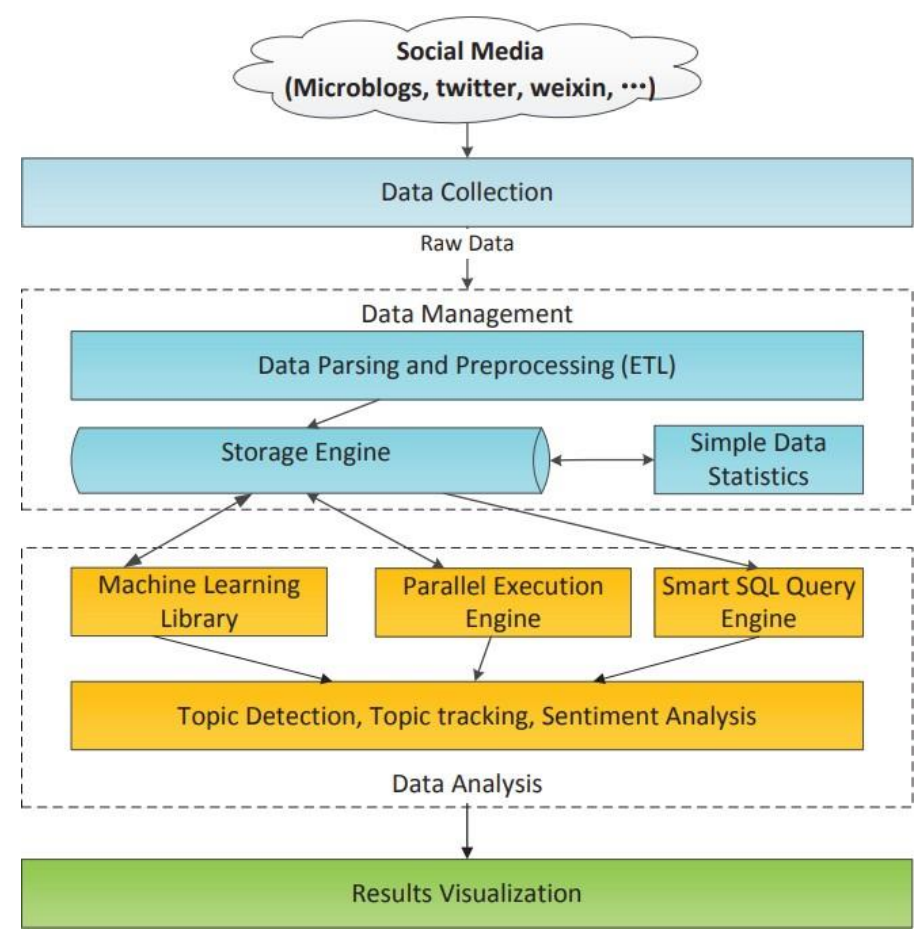

Figure 3: Social Media 
(1) Data Collection: It serves as the foundation for the subsequent analysis. Create a time-line-based micro blogging system.

(2) Real-time Data Management: What we should do with the data that is available is data management. It has data parsing, preprocessing, storage, and basic data statistics.

(3) Data Analysis: We identify micro blogs, extract themes, and evaluate subject evolution, and so on using the preprocessed info.

The fundamental components of the data collecting and analytics framework utilized here are as follows:

1. Interface Server data

2. API Access of data

3. Data Engine

4. Analysis Engine

The Server acts as a conduit for call between the user and the console. Participants in this case0020are internet users, i.e. people who utilize social media websites such as Twitter, and YouTube, among others. Application-specific APIs are used at the API level to connect with a separate Data Engine. These APIs give users access to detailed data components made available. The primary sources collecting engine are social networking site and internet search engines, which provide the analysis engine with a complete dataset. Clustering, classification, text, emotional analysis, and other sorts of experiments are carried out by the analysis engine.

The following are the critical steps of data collection:

1. Identifying the appropriate data source.

2. Identifying the appropriate data type.

3. Choosing the kind of data to be collected.

4. Data storage in a suitable format.

\section{Implementation}

Social networking is a web-based platform that allows users to develop social relationships share information with others via news feeds, opinions, and networking.

\subsection{Using API}

It is possible to create social site data analysis software not only by using web source code, but by working with API given by social site web sites. API is typically (but not always) an abstraction mechanism that differentiates between low and high-level software. The user does not have to analyze social network source code and construct his/her own methods of data retrieval from non-structured sites by utilizing API; instead, it is feasible to utilize existing APIs that give needed data with minimal effort. Open APIs are typically available for public usage, although not everywhere. For example, using the VK programme interface, to obtain all user information, whereas Facebook provides API. The method's advantages include the ability to get user data in a structured format (JSON or XML), also the ease of API calls. 


\section{A. Twitter}

it is a micro blogging website for users to submit messages of up to 140 characters in length. Twitter $4 \mathrm{j}$ is a java library that provides access to the Twitter API. When a developer creates an application using the Twitter API, he or she receives OAuth, which comprises of a key, a secret, an emblem. These used to authorize the user while getting data. To utilise library, the uset have a Twitter account. Once the developer has signed in to his or her Twitter account, he or she may create an application and get authorization. Then, using a platform such as NetBeans, build the java that connects to Twitter over HTTP. Following the creation of the java application, the user should provide the authorization method and get the twitter $4 \mathrm{j}$ to use the needed methods to access the data.

Twitter API has a detriment on the quantity of data that may be retrieved per length of time with each Oauth Knowledge.

Twitter is a service that allows for an amount of posts (tweets). The Twitter API is a that includes techniques for interacting with various data \& managing campaigns. Advertising, Message, and Streaming API is all part of the API interface. The latter two are frequently useful for crawling Twitter data. The Search API allows you to look for popular tweets that have been published in the previous seven days. Because it on rather than completeness, Search API will not return every matched tweets. However, the Search API may limit consequence on many criteria such location. The Streaming API, on the other hand, delivers data over an open, with fresh outcomes provided whenever new matches occur. tweets are triggered by query or a user. the Streaming API give more tweets than the API. The API only give gather tweets that include a certain term inside a specific area. APIs accessed HTTP requests or API libraries written in Python, JavaScript, and other programming languages. The info, however, is available with a token.

EX.need to get stock market-related info from Twitter. There are two algorithms available.

\section{Collect by Group}

We gathered information from three stock market-related organisations. The objective is to develop an application with several classes. One of them has been developed to retrieve all of the users' 338IDs that are members of the groups. Another class was for retrieving data depending on the IDs obtained. The information gathered includes users' screen names, locations, tweets, and eventually the date and time of the incident. The data will be saved in a file for further processing and analysis when it has been retrieved.

The extracted data has been stored in a different file. before executing the application, id were split into 180-id. When the tweets are extracted, each of the 180 people and their associated data will be stored in a file. 


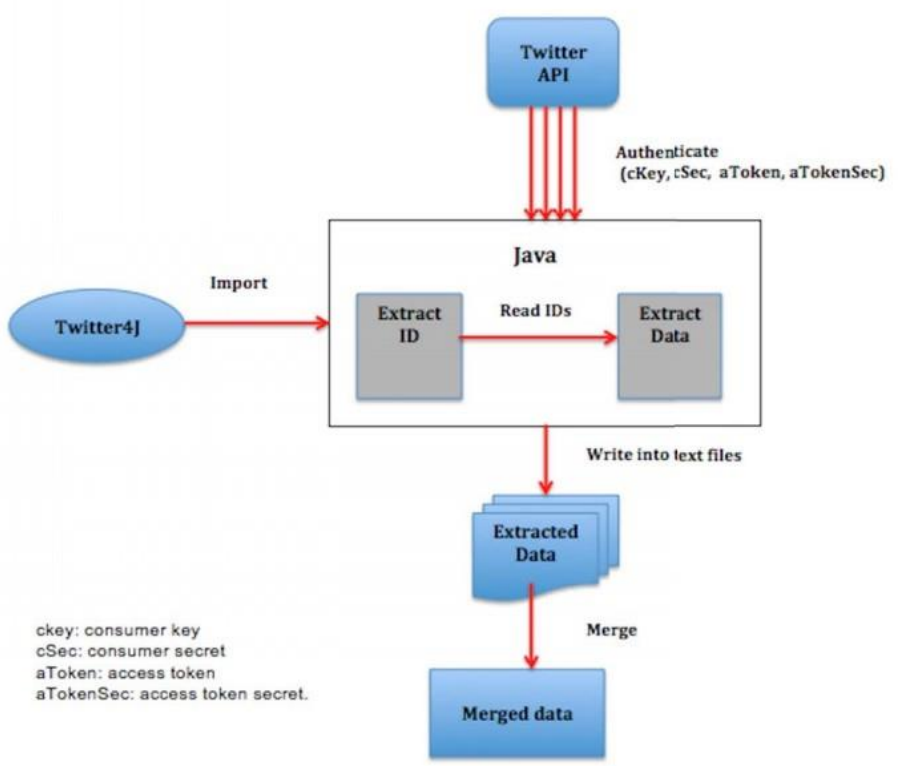

Figure 4: Twitter Data Extraction

COLLECTING DATA FROM DIFFERENT GROUP IN TWITTER

\begin{tabular}{|l||l||l||l|l|}
\hline Group & $\begin{array}{l}\text { Number } \\
\text { of Users }\end{array}$ & $\begin{array}{l}\text { Number } \\
\text { of Tweets }\end{array}$ & $\begin{array}{l}\text { Approximat } \\
\text { time } \\
\text { needed for } \\
\text { collection }\end{array}$ & $\begin{array}{l}\text { End } \\
\text { date of } \\
\text { collection }\end{array}$ \\
\hline StockTwits & 287,720 & $3,282,373$ & 2 months & July 2013 \\
\hline FinancialTimes & $1,700,000$ & $1,576,889$ & 2 months & $\begin{array}{l}\text { October } \\
2013\end{array}$ \\
\hline MarketWatch & 928,066 & $9,909,333$ & Month & $\begin{array}{l}\text { February } \\
2014\end{array}$ \\
\hline
\end{tabular}

Table 1: Data collection from Twitter

\section{B. Filtering}

Method necessitates many process, has identifying the appropriate key for filtering, utilizing them while obtaining from Twitter. The word to take is determined by the study topic. It is connected to the market in our situation. Twitter has a unique symbol for stock, which is a dollar sign followed.

Some terms were discovered on stock twits, a website dedicated to the stock market and Twitter. The keywords picked - "\$YHOO”, "\$P”, “\$EBAY”, "\$BBRY”, "\$TWTR”, "\$V”, "\$YELP”, "\$MDR”, “\$SPLK”, “\$HIMX”, “\$FEYE”, “\$AMZN", "\$NKE", "\$LNKD”.The tweets gathered from May 5th to May 9th is 5367, 1811 users. 


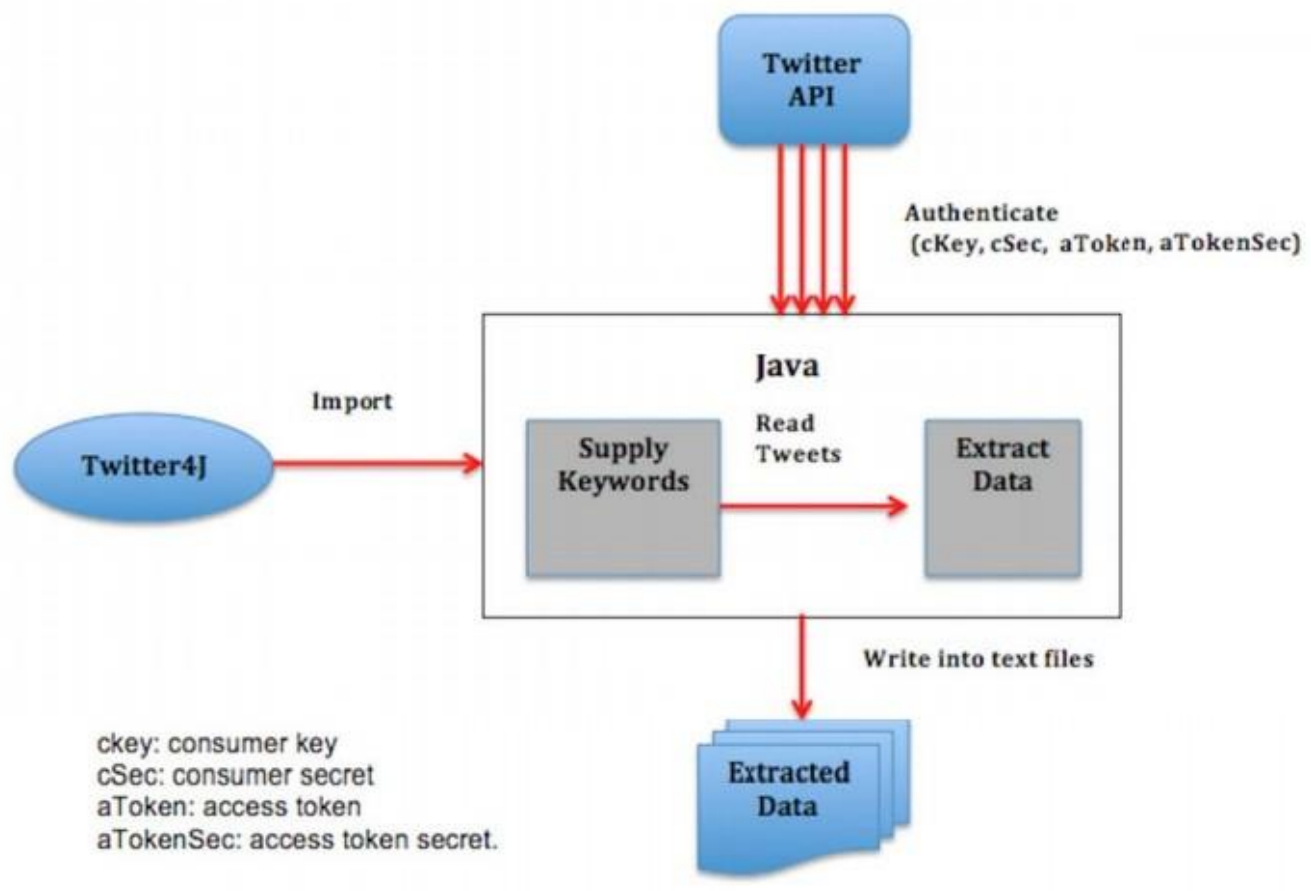

Figure 5: Twitter Data Filtering

COLLECTING DATA BY FILTERING IN MAY 5TH- 9TH

\begin{tabular}{|l||l|}
\hline Keyword & Number of tweets \\
\hline \$YHOO & 299 \\
\hline \$P & 299 \\
\hline \$EBAY & 399 \\
\hline \$BBRY & 664 \\
\hline \$TWTR & 99 \\
\hline \$V & 99 \\
\hline \$YELP & 997 \\
\hline \$MDR & 108 \\
\hline \$SPLK & 237 \\
\hline \$HIMX & 158 \\
\hline \$LBTYA & 245 \\
\hline \$FEYE & 397 \\
\hline \$AMZN & 898 \\
\hline \$NKE & 169 \\
\hline \$LNKD & 299 \\
\hline
\end{tabular}

Table 2: Data Filtering

4.2

Tools Used for Analyzing the Twitter Data 1.Netlytic

It is a text and analyzer that auto summarise huge amounts letter and find social from interactions on sites such as yt and chats. is designed by researchers for researchers, with no programming or API knowledge necessary. Netlytic, you can: 
1. Get (or import) data such as blog comments, forum entries, and SMS conversations, among other things. (For example, create and gather unique data set, or import an data collection.)

2. Identify, investigate emergent topics of conversation to one in your collection.

3. Create, display communica talk tion networks in order for find and get new social site.

Netlytic is suitable for monitoring online interactions in big communities such as classrooms, groups, online reviews forums, and discovered on social media platforms such as yt, blogs, chat rooms and chatting, and etc

\section{Mozdeh}

Gathering tweets matching keywords or from a set of people is (currently) simple using Mozdeh, dependent on Twitter and YouTube's continuing cooperation. Texts from other sources, such as Facebook pages, TripAdvisor, and Scopus, can be imported with extra procedures. Mozdeh is exclusively used to gather text from social media websites.

Mozdeh may collect social web texts directly from websites that distribute them, such as Twitter and YouTube, or import texts acquired in other methods. Twitter and YouTube presently communicate data using an Applications Programming Interface (API), which is an information sharing mechanism with which Mozdeh interacts to download postings. These sites may discontinue or charge for their APIs in the future. The functionality will be removed from Mozdeh in this scenario. As a outcome, gathering data as soon as feasible is a smart idea. Other sites may also begin to provide helpful APIs, which may subsequently be added to Mozdeh.

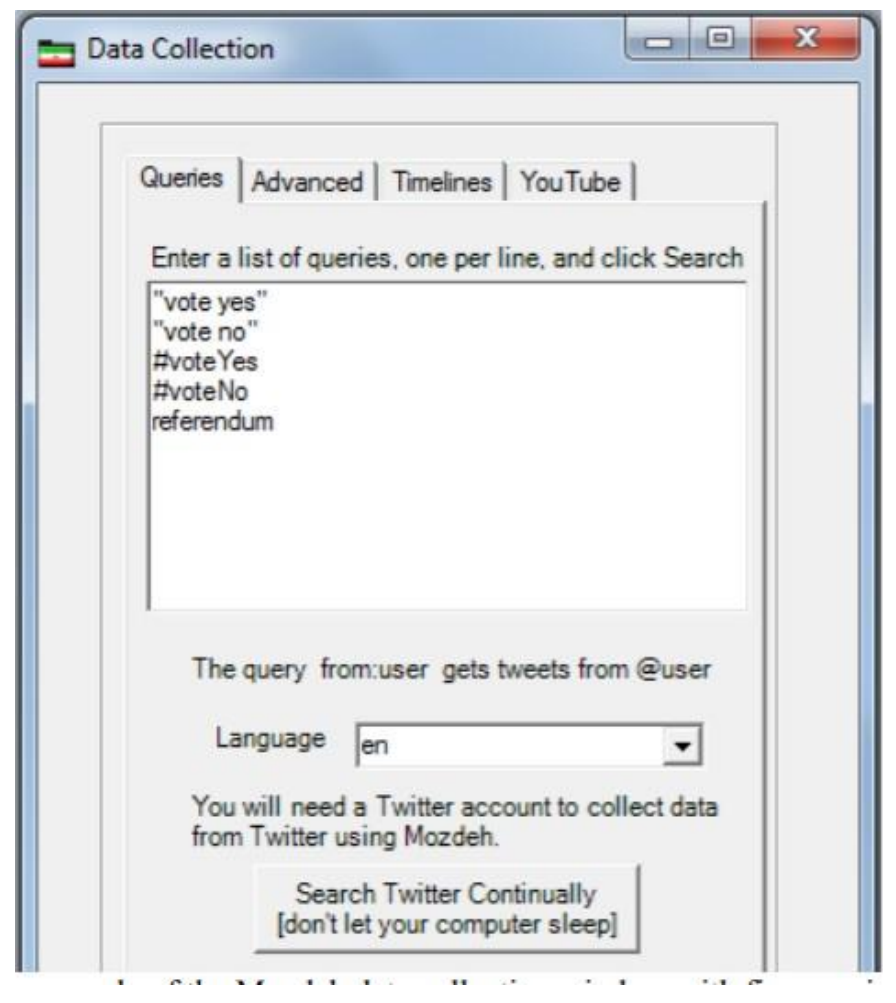

Figure 6: Data Collection 
Example of the Mozdeh data collection window with five queries

Mozdeh may collect tweets that match one or more searches. The data collecting screen contains a list of these queries. When Mozdeh is launched, it iterates over these queries, publishes them to Twitter through its API, and stores the outcomes. This procedure is repeated endlessly to check for fresh content. Mozdeh can gather data for years without ceasing if it is left on a computer that is always connected to the internet and does not enter sleep or hibernation states.

\section{Chorus}

Chorus is, ongoing data harvesting and analytics package meant to help and allow social research utilizing data.

Chorus-TCD is a condition (Tweet Catcher Desktop). It enables you to filter for data in two ways: by discovering a Twitter person and following ones everyday "Twitter lives" (i.e. logical data) or by identifying a network of Twitter people and observing ones everyday "Twitter lifestyles" (i.e. logical data) (i.e. semantically data) (i.e. user data). The interface is made up of three major components. They are located in the upper left corner.

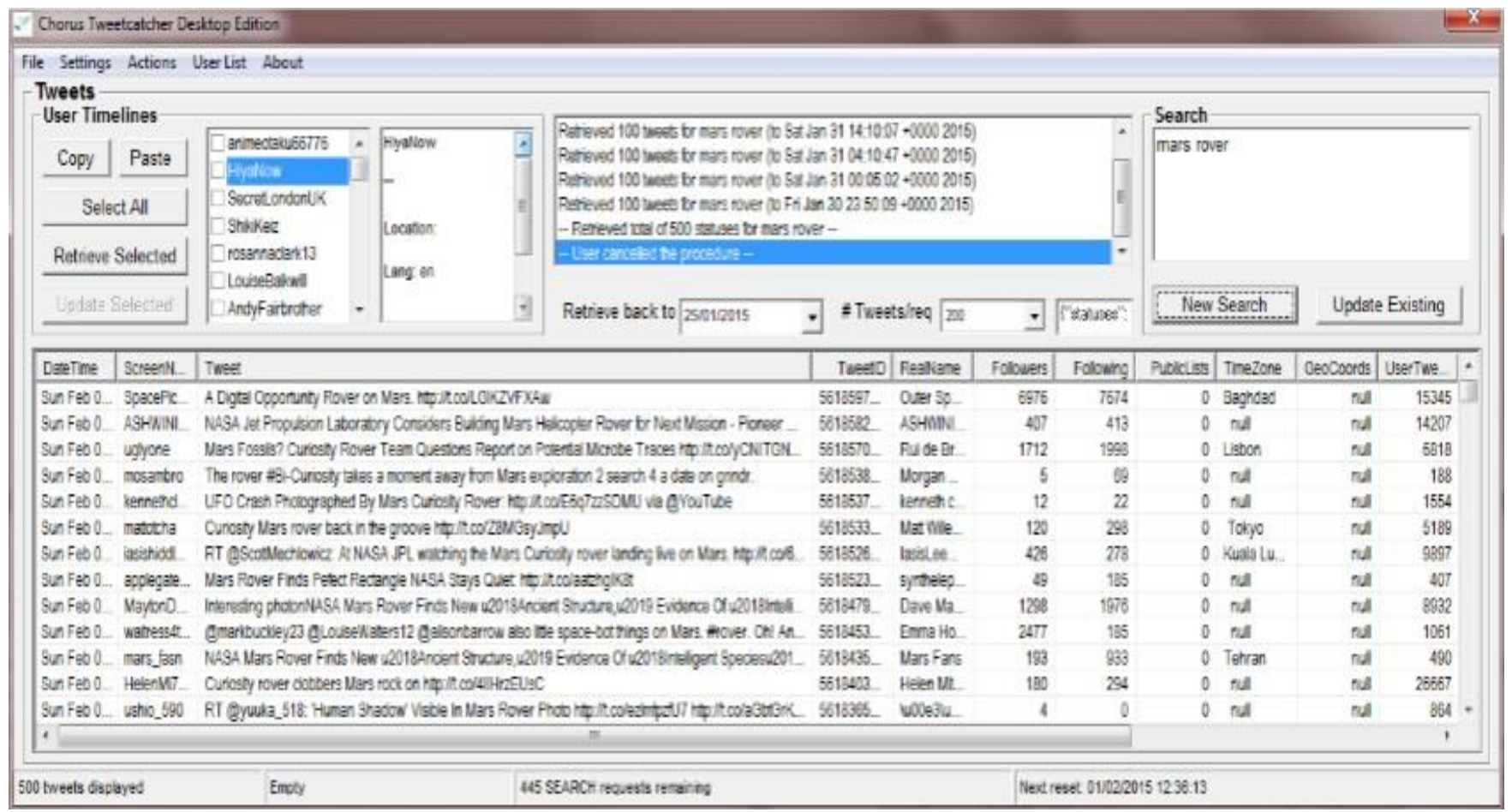

Figure 7: Tweets User Timeline

The controls of getting a query are located in upper right corner. A tabular view of the of any of the aforementioned search modalities may be seen in the bottom half.creates a info file including: 
- Date \&Time

- Screen n Name

- Tweet

- Real n Name

- Followersin

- Followingin

- PublicListsin

- Time of Zone

- Geoco-oords

\section{$5 \quad$ Micro blogging}

Its platforms have grown in popularity and use as a A social networking platform for exchanging information, disseminating knowledge, and finding new connections. People who have signed up for microblogs may submit their thoughts, read other people's posts, leave comments, forward relevant news, and follow their friends. As a outcome, the platform amasses a massive quantity of data.

The main issue is how to acquire the data in an effective and efficient manner. In this part, we offer a data collection approach as well as a time-line tracking methodology.

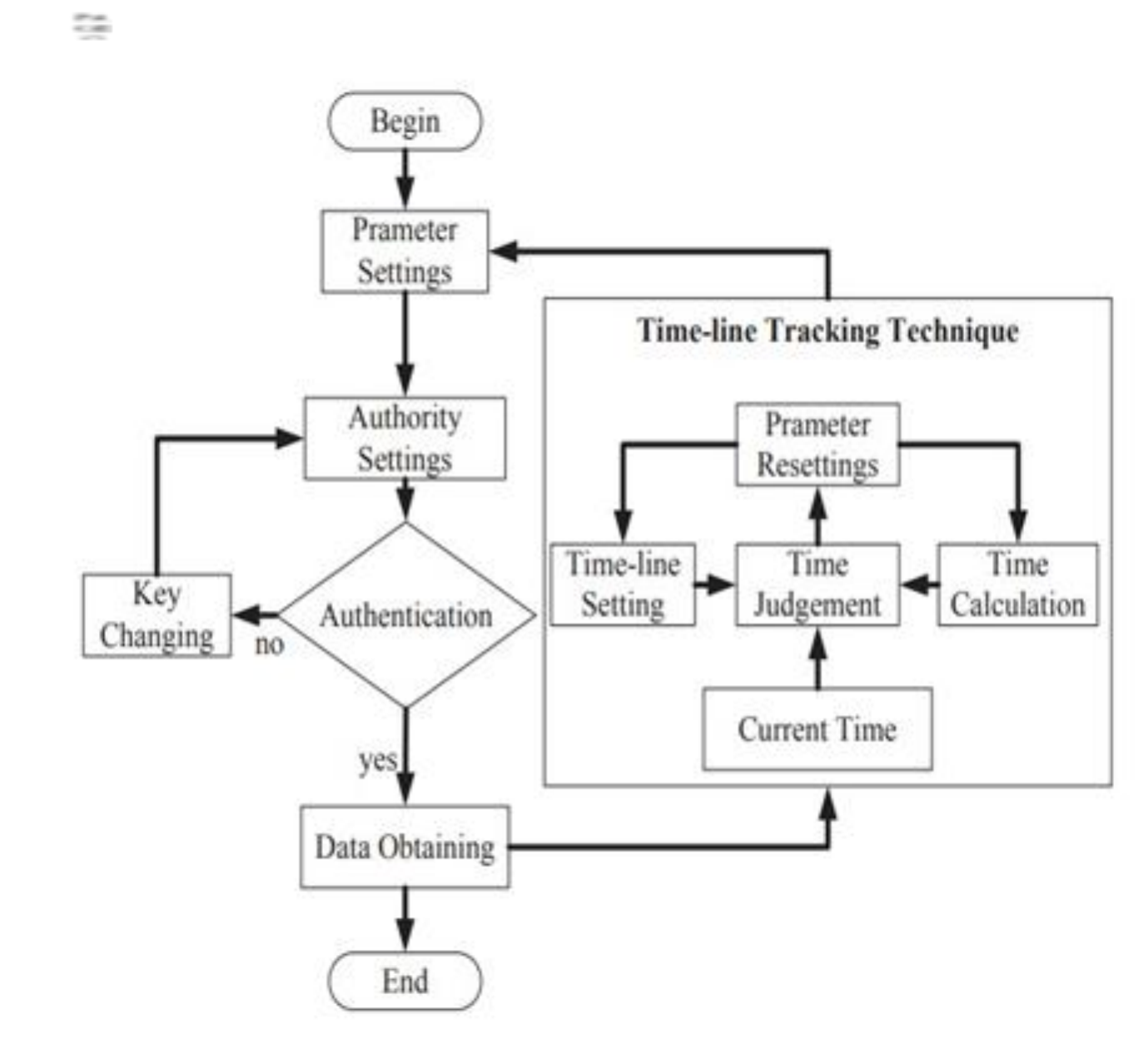

Flowchart 1: Time -Line Tracking Technique 
(1) Parameter Settings: It is made up of two parts. The first relates to the initial different classifiers, such as data type First there aims at establishing the check mark throughout looping, whereas the last refers to inserting the screen capture at both the end of the scrolling operation.

(2) Authority Settings: At this point, create one accounts using the weblog service terminal. Secondly, through authorization, we make a link.

(3) Time-line Tracking Technique: Finally, scientists make decisions out about present point up to a given time setting.

If the timing stamp for the comparisons is sooner than with the data type for the previous time stamp, we use the target time stamp to collect data. Otherwise, we refresh the variable and reset the information and update with the current moment in time mark. We utilize Kafka (http://kafka.apache.org/), a number of different mechanisms programme, in the data gathering module for improve the usefulness of data analysis. This is due to Kafka's growth in output, made splitting, failover, and disturbance when compared to competing encryption methods, making. It is an excellent ideal for developing messaging regulatory compliance. In addition, Kafka can build a user activity monitor funnel as a set of real submit feeds. This is in reference to effectiveness and efficiency of operations (page views, searches, or other activities). Services for those kind of feeds are there for a number of use situations, including real computing, legitimate tracking, including off filtering in Kafka or unavailable business analytics applications.

\section{Scope and Future Work}

We offer a web-based social media data analysis tool that combines qualitative and largescale data mining approaches. We have a lot of work to do in the future. We must complete the implementation of the web services and user interface as intended.

During the process, we must perform user research 387 to determine how we can adapt this social media data analysis tool to fit the needs of various research objectives, as well as which features we should add or delete. We will also broaden the data coverage to include additional social media platforms than Twitter. We will perform usability testing after we have a pretty full functioning prototype in order to discover usability issues and further develop the product. As indicated in the database implementation, we will experiment with NoSQL databases based on Bigtable, such as Accumulo, to further enhance the speed of this webbased application, because they are often more flexible at handling web-scale data. Another possible future effort would be to employ topic analysis to propose various categories, easing the strain on researchers while analyzing large-scale data.

\section{Conclusion}

Materials is a major stage in every work or project since data is the major element we use for exploration as evaluation. Photo sharing networks are a fantastic source of information. As a result, using the appropriate API lengthens the calculation. In this post, we discussed many methods for obtaining twitter api. The aim was to make money in the process. One strategy began with either the group and progressed to the recruitment of users. The else technique get 
with a key phrase but just discovered relevant tweets, recruiting individuals to build a population sample.

Each approach has a different advantage. As a result, area by users may be accessed and abused in the same manner that hand tools or specialised software can. Social network assessment is used in a variety of programmes and disciplines. Data storage and management, analysis of distinguishing features and user activity, and messaging are some of the applications.

\section{References}

1. M. T. Schafer and K. van Es, "Social data," in " The datafied society: Studying culture through data. Amsterdam University Press, 2017, ch. 10, pp. 147-154.

2. A. M. Kaplan and M. Haenlein, "Users of the world, unite! the challenges and opportunities of social media," Bus. Horizons, vol. 53, no. 1, pp. 59-68, 2019.

3. P. Gundecha and H. Liu, "Mining social media: a brief introduction," in New Directions in Informatics, Optimization, Logistics, and Production. Informs, 2012, pp. 1-17.

4. Z. Tufekci, "Big questions for social media big data: Representativeness, validity and other methodological pitfalls." ICWSM, vol. 14, pp. 505- 514, 2014.

5. D. Ruths and J. Pfeffer, "Social media for large studies of behavior," Science, vol. 346, no. 6213, pp. 1063-1064, 2014.

6. Chen and D. B. Neill, "Human rights event detection from heterogeneous social media graphs," Big Data, vol. 3, no. 1, pp. 34-40, 2015.

7. M. De Choudhury, "Anorexia on it: A characterization study," in Proceedings of the 5th International Conference on Digital Health 2015. ACM, 2015, pp. 43-50.

8. Chengang Zhu, Guang Cheng (senior member, IEEE) and Kun Wang (senior member, IEEE), "Big data analytics for program popularity prediction in broadcast TV industries", IEEE Access, October'2017.

9. MohammadNoor Injadat, Fadi Salo, Ali Bou Nassif, "Data mining techniques in social media: A survey", Neurocomputing- ScienceDirect, June'2016.

10.Marouane Birjali, Abderrahim Beni-Hssane, Mohammed Erritali, "Analyzing social media through big data InfoSphere BigInsights and Apache Flume", ScienceDirect, June'2017.

11.KuaiXu ,Feng Wang, Haiyan Wang, and Bo Yang "Detecting Fake News Over Online Social Media via Domain Reputations and Content Understanding" TSINGHUA SCIENCE AND

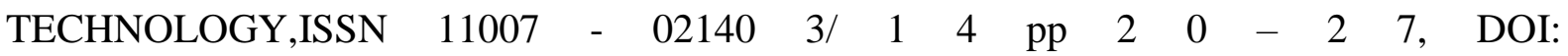
10.26599/TST.2018.9010139, Volume 25, Number 1, February 2020.

12.TAO JIANG, JIAN PING LI, AMIN UL HAQ, ABDUS SABOOR, AMJAD ALI,"A Novel Stacking Approach for Accurate Detection of Fake News" Digital Object Identifier 10.1109/ACCESS.2021.3056079. 
13.MUHAMMAD UMER, ZAINAB IMTIAZ, SALEEM ULLAH, ARIF MEHMOOD, GYU SANG CHOI, BYUNG-WON ON "Fake News Stance Detection Using Deep Learning Architecture (CNN-LSTM)" Digital Object Identifier 10.1109/ACCESS.2020.3019735.

14.XINYI ZHOU, REZA ZAFARANI,"A Survey of Fake News: Fundamental Theories, Detection Methods, and Opportunities" ACM Comput. Surv.1, 1, Article 1 (January 2020), 37 pages.

15.Shivani S Nikam, Prof.RupaliDalvi, " Machine Learning Algorithm based model for classification of fake news on Twitter", 2020 Fourth International Conference on I-SMAC (IoT in Social, Mobile, Analytics and Cloud) (I-SMAC),DOI: 10.1109/I-SMAC49090.2020.9243385.

16.Anand.RPushpalatha.M DrRajshekhar M Patil, “A Social Networking for Sharing Infrastucture Resources in the Social Cloud Computing", International Journal of Informative \& Futuristic Research (IJIFR),2016.

17.Dr. M V VijaykumarJagadish P, Shryavani K , Anand R, “Authorized Deduplication in Hybrid Cloud”, IJCSN International Journal of Computer Science and Network,2016.

18.Mr.Anand.RPriyanka Dr. Rajshekhar M. Patil, "Health Monitoring In Aerospace System”, International Journal of Informative \& Futuristic Research (IJIFR), 2017.

19.Anand.R Dr. Deepak S Sakkari "Twitter Data Collection Using Tools for Classification of Fake Data", STRAD Research, Volume 8, Issue 8, August - 2021, 495-505.

20.Deepak. S. Sakkari, T. G. Basavaraju, "GCCT: A Graph-based Coverage and Connectivity Technique for Enhanced Quality of Service in WSN", WPC-Springer, December 2015, Volume 85, Issue 3, pp 1295-1315

21.Deepak. S. Sakkari, T. G. Basavaraju, "Energy efficient scheme to Jointly Optimize Coverage and Connectivity in Large Scale Wireless Sensor Network", IJECE (SCOUPUS Indexed), Vol. 5, No. 3, June 2015, pp. 454 463

22.Mohammed Mujeerulla, Deepak S Sakkari "Design and implementation of elliptic curve digital signature using bit coin curves secp256k1 and secp384r1 for base 10 and base16 using Java", International Conference on Innovation in Electrical Power Engineering, Communication, and Computing Technology (IEPCCT 2021), Springer Conference, 25th September 2021. 